\title{
Estimating the frequency and cost of emergency department presentations and hospitalisation of COPD patients: A retrospective analysis from regional Queensland
}

\begin{abstract}
Objective: Chronic Obstructive Pulmonary Disease (COPD) patients commonly have frequent visits to the Emergency Department (ED). Consequently, COPD has a significant effect on total healthcare expenditure.
\end{abstract}

The objective was to measure the frequency of ED presentation and hospitalisation among COPD patients and to estimate the costs resulting from such care utilisation.

Methods: This was a causal-comparative non-experimental research design conducted in three regional hospitals between 2016 and 2018. Two different original data sets were used: an automated hospital data set and an audit of patient charts. Secondary cost data were also used. Data were analysed using Pearson's chi-square test to estimate the relationship between several patient and treatment-related characteristics.

Results

There were 5,253 patient presentations at ED and hospital length of stay data were available for 5,079 COPD patients. The total cost of hospital stays was $\$ 42.14$ million for the time period and the mean average cost was $\$ 8,297$ for ED patients and were admitted to hospital. Factors significantly associated with hospital length of stay were age and time spent in the ED. Noticeably, one in two (51.7\%) COPD patients were discharged from ED (all destinations) within 4 hours irrespective of their triage category.

Conclusions: COPD patient presentation to ED and admission to hospital is an expensive method of providing health care to manage this chronic condition. Clinical practitioners and policy makers need to develop and implement optimal integrated care management systems to reduce this hospitalisation rate and reduce the societal costs associated with COPD patient management.

\section{Keywords:}

COPD, Regional, Queensland, Emergency Department, Costs 


\section{Introduction}

Chronic Obstructive Pulmonary Disease (COPD) is a common and progressive disease, and one of the principal causes of chronic morbidity and mortality, globally. According to the Global Burden of Disease Study report from the World Health Organisation (WHO) the prevalence of COPD reached 251 million cases in 2016 and approximately 3.17 million deaths were associated with COPD globally in $2015^{1}$; with aging populations the number of COPD cases is expected to increase by more than $30 \%$ over the next decade ${ }^{1}$. COPD is an irreversible and debilitating lung condition with airflow limitations $s^{2,3}$ and patients often suffer from limited mobility and frailty, an inability to work and reduced quality of life, as well as frequent hospital visits $^{4,5,6}$. This has a significant cumulative effect on total healthcare expenditure (both inpatient and outpatient care) and employment-related costs (loss of productivity) ${ }^{7,8}$.

The prevalence of COPD in Australia was 1 in 20 (population aged 45 or over) in 2017-18 and was the $5^{\text {th }}$ leading causes of mortality (7518 deaths) in $2017^{9}$. The COPD mortality rate has declined in Australia over the last decade however the rate of COPD-related hospitalisation has increased from 718 people in $2007-08$ to 757 in $2016-17$ per 100,000 population (age $\geq 45)^{10}$. According to the Australian Institute of Health and Welfare (AIHW), the total number of patients who were subsequently admitted (presentations included all visit types, i.e. the episode end status was 'Admitted to this hospital') after emergency department (ED) presentation with COPD (principal diagnosis code J44) in 2017-18 was equal to 34,569 inpatients with an average length of stay of five days ${ }^{11}$. In 2015-16, the Australian health system spent an estimated $\$ 976.9$ million treating COPD patients, which is $24 \%$ of expenditure on all respiratory conditions ${ }^{9}$. Hospitalisation comprises between $52 \%$ to $84 \%$ of direct healthcare expenditure related to $\mathrm{COPD}^{12,13,14}$. Understanding key socio-demographic characteristics of frequently presenting patients could reduce the healthcare cost burden and optimise healthcare usage $^{15}$.

There is a scarcity of literature which has studied the demographic characteristics of COPD patients who present to EDs and are subsequently admitted as inpatients to hospital. As ED presentations, hospitalisation and length of stay are easy to observe; these factors of presentation can be effective for estimating trends in COPD-related health care utilisation and the direct costs associated with them ${ }^{16}$. Australia had one of the highest rates of COPD-related hospitalisations of OECD countries in $2015^{17}$. Carefully planned self-care management and integrated care have the potential to substantially reduce ED presentations and hospitalisations ${ }^{18}$ and therefore reduce COPD treatment costs. 
The study objective was to measure the frequency of ED presentation and hospitalisation of COPD patients and to estimate the costs resulting from such health care utilisation using hospital-based data between 2016 and 2018. The demographic characteristics and triage categories of COPD patients in three hospitals in Queensland, Australia were also identified.

\section{Methods}

\section{Design and setting}

This was a causal-comparative non-experimental research design conducted in three hospitals in the same geographical region with one hospital (Gold Coast) a 750-bed tertiary referral centre and the other two being the main hospitals in their areas.

\section{Study population}

During the 20-month study period there were a total of 5,365 patient presentations to ED which had a primary medical diagnosis code of J44 (COPD). Those patients with COPD but who were admitted with an alternative diagnosis code were not included in the study. Australian Triage Scale (ATS) is ranked from category 1 (a life-threatening condition) to category 5 (minor illness). These COPD patients were at the main hospitals of three Hospital and Health Services: Toowoomba Hospital (Darling Downs), Ipswich Hospital (West Moreton) and Gold Coast University Hospital (Gold Coast). Patients who were discharged from ED to home or usual residence and stayed at the hospitals (after ED) less than one day were considered as zero or no hospital stay.

\section{Data collection}

This causal-comparative non-experimental research design included two distinct original data sets. The first was an automated data set extracted from the EDIS and HBSCS systems of admission data for every ED presentation with COPD as the primary diagnosis code. The second dataset consisted of a random clinical file chart audit of 40 patient files (from the study cohort) from each of three sites $(n=120)$ to assist with causal analysis. Secondary data on costs were obtained from the National Health Performance Authority (NHPA) ${ }^{2}$, Independent Hospital Pricing Authority (IHPA) ${ }^{22}$ and the Queensland Ambulance Service (QAS) ${ }^{19}$. The IHPA report collected the average cost from 451 public hospitals for admitted acute care. Admitted acute care is provided to admitted patients to receive active but short-term care with the objective of cure, relieve symptoms or injury, reduce illness severity, perform surgery etc. According to the IHPA report, the average cost of admitted acute separations (selected conditions including COPD) in Australian hospitals for patients who were admitted and 
discharged in the same financial year (2016-17) was $\$ 5,229$, and the mean LOS was 2.53 days. Hence, the average cost of each day in hospital was calculated as $\$ 2,066(\$ 5,229 / 2.53)^{22}$.

In addition, the average cost (per episode) of emergency presentation in 2016-17 was $\$ 666$ in Australia $^{22}$. However, in Queensland, the average cost of an admitted ED presentations was $\$ 940$, and non-admitted presentations was $\$ 524^{22}$. Hence, the weighted average cost (WAC) of all ED presentations among COPD patients included in this study was $\$ 795.65$ (WAC $=$ $\$ 940 \times 65.3 \%+\$ 524 \times 34.7 \%$ ), as $65.3 \%$ of them had two or more days hospitalisation. Here, the assumption is that those patients who were discharged from hospital (after ED presentation) within one day or less, were non-admitted patients.

The QAS report estimated the cost of ambulance services for emergency, urgent, nonemergency, and medically authorised incidents ${ }^{19}$. The average cost per incident (all patients in 2016-17) was \$568, \$854, \$591 for Gold Coast, Darling Downs and West Moreton area, respectively. The 2016-17 road ambulance budget and road ambulance activity were used in calculating the mean cost per incident.

\section{Model and Statistical Analysis}

Initially a Pearson's chi-square test was used to estimate the relationship between triage category and several key patient and treatment-related characteristics. The test is conducted to measure the relationship between two categorical variables. The primary aim of the test is to compute variations among the pattern of observed frequencies from the pattern of expected frequencies ${ }^{20}$.

Next, a generalised linear model (bivariate regression with a maximum likelihood estimate) was used to determine the factors that influence the LOS among COPD patients. The generalised linear model (GLM) combines the systematic and random components of the linear model in a consistent way.

Next, the independent sample $t$-test estimate was calculated to examine the relationship between the number of ED presentations and several key independent variables. This test is widely used to calculate the mean differences between different participants. A major assumption of this test is that each subgroup of participants should be unrelated, and the groups come from a population with equal variances ${ }^{20}$.

Finally, a binary logistic regression model was constructed to examine the relationship between multiple ED presentations and distance to the hospital. Logistic regression is used to predict a categorical variable from a set of predictor variables ${ }^{21}$.

\section{Ethics Approval}


Multisite ethics approval was obtained from Queensland Health (HREC/17/QGC/249).

\section{Results}

\section{Descriptive statistics}

A total of 5365 presentations occurred in the three hospitals during the period late-2016 to late2018. The mean age of these patients was 71 years, median triage category was three (urgent), on average they spent 4 hours and 40 minutes in ED, around $90 \%$ had no private health insurance, less than 10\% were currently employed, and $74 \%$ were born in Australia. Among these patients, 58\% had more than one COPD related ED presentation during this period. Data regarding hospital admission and LOS were available for 5079 patients out of which 1006, 1715 and 2358 were from Toowoomba, Ipswich and Gold Coast Hospital, respectively. Approximately, $34 \%$ had one or fewer days of hospital LOS.

Table 1 depicts the link between triage categories and time spent at ED, as well as triage category and first point of contact (who referred by) of COPD patients. Time in ED varied significantly across triage categories, with ATS 1 (a life-threatening condition) being associated with a higher number of hours spent in the ED (p-value<0.05). Notably, 51.7\% COPD patients received treatment and were discharged from ED (all discharge destinations) within 4 hours irrespective of their triage category.

\section{<Table 1 here>}

A majority $(82.5 \%)$ of the presentations were referred to the ED through self/family/friend recommendation (the first point of contact is ED staff) with a small minority (7\%) came through GP referral. More than $85 \%$ of patients with triage category 1 (life-threatening), and 2 (very urgent) who presented were referred by self, family or friends.

\section{<Table 2 here>}

Life-threatening and very urgent conditions (ATS 1 and 2) were most likely to have more than five-day hospital LOS (Table 2). One in three COPD patients who came into ED were not admitted to hospital. In contrast, $18.5 \%$ of COPD patients had $>5$ days LOS.

COPD related ED presentation significantly differs due to age (p-value $<0.05)$ and higher age was associated with a higher number of ED presentations across all triage categories (analysis not shown). Similarly, hospital LOS was also significantly related to age and increasing age was associated with a higher hospital LOS ( $p$-value<0.05) (analysis not shown). $19.8 \%$ of COPD patients who were admitted to hospital and aged over 65 had more than five days LOS. 


\section{Cost of ED presentation and hospitalisation}

Table 3 shows the total and average cost of ED presentation and ambulance services for COPD patients. This table exhibits the total cost, as well as the cost to each hospital, separately. Table 3 also shows patients and their LOS for individual hospitals. For simplification the average cost per ED presentation and per day LOS for admitted acute separations in Australian hospitals was used to calculate the total cost of ED presentations and hospitalisation ${ }^{2}$.

Approximately $\$ 4.18$ million of health care resources were utilised during the study period to treat patients with COPD at the ED. The annual cost for the period of late 2017-18 (with 2440 ED presentations) was $\$ 1.94$ million (2440 x \$795.65). Noticeably, around \$2.5 million were spent on patients who experienced multiple ED presentations.

$<$ Table 3 here>

Table 3 also provides information regarding the cost of ambulance services to transport COPD patients to the hospital. The ambulance services cost was collected from information provided by QAS $^{19}$. During the study period, 4246 COPD patients used this service, and the total cost was around \$2.79 million.

The total cost of hospital stay is presented in Table 4. The results indicate that hospital LOS data were available for 5079 COPD patients who presented to the ED. The total cost of their hospital stay was $\$ 42.14$ million during the time period of 2016-2018. The mean average cost was $\$ 8297$, and the 5\% trimmed mean (removing extreme values) was $\$ 6932$. Furthermore, the annual cost of hospital admissions was $\$ 19.82$ million with a mean of $\$ 8122$, with a trimmed mean value of $\$ 6812$. Confirmatory data comparisons stemming from an international pool of evidence in available literature demonstrated similar findings. Dang-Tan et al. ${ }^{18}$ concluded the average cost from a societal perspective was CAN\$3,910-6,693; Foo et al. ${ }^{12}$ estimated the cost to be US\$9,981 for the USA, US $\$ 4,650$ for Japan and US\$3,224 for the UK. Using the conversion rate ( $\mathrm{A} \$ 1=\mathrm{US} \$ 0.74$ in 2016), the average cost per admission in the hospitals in Queensland was US\$6,010 (\$8,122 x 0.74). Furthermore, according to the NHPA2 the average cost per hospital admission for COPD patients in 2011-12, ranged from $\$ 3,600$ to $\$ 7,800$. Considering the growth of cost for treating patients in hospital from 2011-12 to 201718 , the estimate of $\$ 8,122$ falls well within the established range and was comparable to costings stemming from international data ${ }^{12,18}$.

<Table 4 here> 
$<$ Table 5 here>

Table 5 shows the key risk factors associated with longer duration hospital LOS. Older patient age and greater time spent in the ED were significantly associated. A one-year increase in age was associated with 0.036 days of additional LOS ( $p$-value<0.05). Similarly, one additional hour spent at ED was associated with an increased LOS of 0.116 days ( $p$-value<0.05). Employed patients had significantly less LOS ( $p$-value<0.05). Patients with ATS 1 were significantly more likely to have extended LOS $(p$-value $<0.05)$. Private health insurance status and country of birth (Australia versus overseas) were not associated with LOS.

\section{Discussion, Study Limitations and Conclusions}

The study objective was to measure the frequency of ED presentation and hospitalisation among COPD patients and to estimate the costs resulting from such care utilisation. This study provides contemporaneous insight into the frequency, demographic characteristics and cost of COPD patients presenting to ED in three Queensland hospitals. The study is limited to the southern region of Queensland and therefore one State within Australia. The study is also restricted to regional hospitals only. Broader confirmatory research including metropolitan and rural and remote EDs is still required to consider the transferability of findings. The study shows that COPD-related ED presentations and hospitalisations were significantly and strongly related to increasing age of patients. Previous studies have concluded that the risk of hospitalisation among COPD patients increases in conjunction with age and comorbidities ${ }^{23,24}$. Hence, as COPD patients grow older, and with increased comorbidities, the likelihood of ED presentation and increased LOS after admission increases significantly. Although, $48 \%$ of Australians held private health insurance cover ${ }^{25}$; nonetheless, in this study cohort, around $90 \%$ of patients did not and solely relied upon public health care. This situation imposes additional burdens on the financing of public healthcare in Australia.

The average LOS in ED in Queensland hospitals across all presentations in 2017-18 was 6 hours and 48 minutes $^{11}$. However, the average LOS at ED in this study was 4 hours and 40 minutes. Queensland wide data showed that $88.7 \%$ of COPD patients presented at ED were triage Category 3 or higher but here was $97.1 \%$. Extended time in the ED has a significant and positive correlation with longer hospital inpatient LOS. It seems that patients who stay longer in $\mathrm{ED}$ are triage 1 and 2 and due to the severity of their conditions, they were subsequently admitted and had prolonged LOS. 
Eight out of 10 COPD patients were referred by self or family or friends with only $7 \%$ being referred by general practitioners. This large number of unplanned admissions places more pressure on EDs that are already experiencing high caseloads. This situation significantly increases the costs of treating COPD patients who could be diverted into less expensive treatment modes (stepped care) especially within primary health care. Although, primary care professionals have been given significant responsibility and autonomy to treat COPD patients $^{24}$, prevalence of comorbidities ${ }^{26}$, ineffective self-care management and the lack of integrated COPD care programs and system responses ${ }^{17}$ often increase the overall probability of an ED visit and subsequent hospital admission. The research also shows a range of reported gaps in GP capacity, capability and willingness to provide thorough management of COPD in the primary care setting, including a lack of appropriate knowledge, skills, confidence and education to provide support to patients ${ }^{27}$. Past research has concluded that COPD is often underdiagnosed (failing to identify early symptoms), and delays in diagnosis mostly result in unnecessary (avoidable) utilisation of healthcare resources especially within $\mathrm{ED}^{26}$. Therefore, policies to better train and support primary care professionals on the signs and symptoms of COPD to assist early diagnosis ${ }^{25}$ are necessary.

The cost estimates of ED presentations and hospital LOS are negatively influenced by the significant number of patients who had comorbidities (treatment levels varied markedly), and by the use of average cost values. However, it is assumed that ED and other hospital services for COPD patients, specifically with acute exacerbations, will consume more resources than a general ED presentation or a primary health care consultation. There is an expectation that comorbidities will have an influence on presentations and costings yet data to investigate this issue were not available. This is a significant limitation of this study that should be explored in subsequent research.

Therefore, it can be concluded that the study results provide a comparable illustration of the costs of hospitalisation of COPD patients in three regional hospitals in Queensland, Australia. Increased awareness of the relevant characteristics and trends, along with the cost of care of COPD patients presenting to ED (often more than once) and their hospital admission (often multiple times) will assist practitioners and policymakers in developing more optimal and resource-effective integrated care management models which could reduce the hospitalisation rate and reduce the societal cost of managing COPD patients. Increased community screening leading to earlier diagnosis of COPD coupled with increased investment in primary care disease optimization strategies is likely to reduce the frequency of acute exacerbations, ED 
presentations, and lead to a reduction in functional decline thereby optimising disease management.

The frequency and cost of system utilisation is significant for COPD patients who present to ED rather than being managed through primary care and we now have baseline characteristics and costs against which alternative strategies for this patient group might be compared. 


\section{References}

1. World Health Organization (WHO), (2020). Chronic respiratory diseases - Burden of COPD. Accessed on 6 February 2020 from https://www.who.int/respiratory/copd/burden/en/

2. National Health Performance Authority (NHPA), (2015). Hospital Performance: Costs of acute admitted patients in public hospitals in 2011-12. https://www.myhospitals.gov.au/ourreports/cost-of-acute-admitted patients/april2015/pdffiles/hp_costsofacuteadmittedpatients_2011_12_rpt.pdf

3. Darnell, K., Dwivedi, A., Weng, Z. et al. (2013). Disproportionate utilization of healthcare resources among veterans with COPD: a retrospective analysis of factors associated with COPD healthcare cost. Cost Effectiveness and Resource Allocation, 11:13.

4. Wheaton A., Cunningham T., Ford E. et al. (2015). Employment and activity limitations among adults with chronic obstructive pulmonary disease - United States, 2013. MMWR. Morbidity and Mortality Weekly Report, 64(11):289-295.

5. Singh, J., and Yu, S. (2016). Utilization due to chronic obstructive pulmonary disease and its predictors: a study using the U.S. National Emergency Department Sample (NEDS). Respiratory Research, 17:1.

6. van Boven, J., Vegter, S, van der Molen, T. et al. (2013). COPD in the working age population: the economic impact on both patients and government. COPD, 10(6):629-639.

7. Patel, J., Nagar, S., and Dalal, A. (2014). Indirect costs in chronic obstructive pulmonary disease: a review of the economic burden on employers and individuals in the United States. International Journal of Chronic Obstructive Pulmonary Disease, 9(1):289-300.

8. Kim, J., Lee, T., Kim, S. et al. (2016). The economic burden of chronic obstructive pulmonary disease from 2004 to 2013. Journal of Medical Economics, 19(2):103-110.

9. Australian Institute of Health and Welfare (AIHW), (2019). Chronic obstructive pulmonary disease (COPD). Cat. no. ACM 35. Canberra: AIHW. Accessed 6 February 2020 from https://www.aihw.gov.au/reports/chronic-respiratory-conditions/copd

10. Australian Institute of Health and Welfare (AIHW), (2014). Mortality from Asthma and COPD in Australia. Cat. no. ACM 30. Canberra: AIHW. https://www.aihw.gov.au/getmedia/62e7a82f-56f1-4dad-86b0$\underline{\text { f65d5bc8d74b/17476.pdf.aspx } \text { ?inline }=\text { true }}$ 
11. Australian Institute of Health and Welfare (AIHW), (2018). Emergency department care 2017-18: Australian hospital statistics. Health services series no. 89. Cat. no. HSE 216. Canberra: AIHW.

12. Foo, J., Landis, S., Maskell, J., et al. (2016). Continuing to Confront COPD International Patient Survey: Economic Impact of COPD in 12 Countries. PLoS One, 11(4):e0152618.

13. ur Rehman, A., Hassali, M., Muhammad, S. et al. (2019). The economic burden of chronic obstructive pulmonary disease (COPD) in the USA, Europe, and Asia: results from a systematic review of the literature. Expert Review of Pharmacoeconomics \& Outcomes Research, DOI: 10.1080/14737167.2020.1678385.

14. Seifer, F., Hansen, G. and Weycker, D. (2019). Health-care utilization and expenditures among patients with comorbid bronchiectasis and chronic obstructive pulmonary disease in U.S. clinical practice. Chronic Respiratory Disease, 16:1-8.

15. Shah, T., Churpek, M., Perraillon, M. and Konetzka, T. (2015). Understanding why patients with COPD get readmitted. Chest, 147(5):1219-1226.

16. Organisation for Economic Co-operation and Development (OECD), (2017). Health at a Glance 2017: OECD Indicators. Accessed on 6 February 2020 from http://dx.doi.org/10.1787/health_glance-2017-en

17 Souliotis, K.. Kousoulakou, H., Hillas, G., Tzanakis, N., Toumbis, M., Vassilakopoulos, T. (2017). The direct and indirect costs of managing chronic obstructive pulmonary disease in Greece. Int J Chron Obstruct Pulmon Dis, 12:1395-1400

18. Dang-Tan, T., Ismaila, A., Zhang, S. et al. (2015). Clinical, humanistic, and economic burden of chronic obstructive pulmonary disease (COPD) in Canada: a systematic review. BMC Research Notes, 8:464.

19. Queensland Ambulance Service (QAS), (2018). Public performance indicators - financial year to date - 2017-2018. Accessed on 6 February 2020 from https://www.ambulance.qld.gov.au/docs/QAS-QTR-3-Public-Performance-Indicators-FY1718.pdf

20. Field, A., (2013). Discovering statistics using IBM SPSS statistics. 5th edition. Sage, London.

21. Harrell, F. (2015). Binary Logistic Regression. pp. 219-274 in Regression Modeling Strategies. Springer, Amsterdam. 
22. Independent Hospital Pricing Authority (2019). National Hospital Cost Data Collection Report: Public Sector, Round 21 Financial Year 2016-17. Canberra. Accessed 6 February 2020 from:

https://www.ihpa.gov.au/sites/default/files/publications/national_hospital_cost_data_collectio n_australian_public_hospitals_cost_report_round_21_2016-17.pdf

23. Germini, F., Veronese, G., Marcucci, M. et al. (2018). COPD exacerbations in the emergency department: Epidemiology and related costs. A retrospective cohort multicentre study from the Italian Society of Emergency Medicine (SIMEU). European Journal of Internal Medicine, 51:74-79.

24. May, S., and Li, J. (2015). Burden of chronic obstructive pulmonary disease: healthcare costs and beyond. Allergy and Asthma Proceedings, 36(1):4-10.

25. Australian Institute of Health and Welfare (AIHW), (2019). Chronic obstructive pulmonary disease (COPD). Cat. no. ACM 35. Canberra: AIHW. Accessed 6 February 2020 from https://www.aihw.gov.au/reports/chronic-respiratory-conditions/copd

26. Mannino, D., Higuchi, K., Yu, T-C. et al. (2015). Economic burden of COPD in the presence of comorbidities. Chest, 148(1):138-150.

27. Russell, S., Ogunbayo, O., Newham, J., Heslop-Marshall, K., Netts, P., Hanratty, B., Beyer, F., and Kaner, E. (2018). Qualitative systematic review of barriers and facilitators to selfmanagement of chronic obstructive pulmonary disease: views of patients and healthcare professionals. NPJ Primary Care Respiratory Medicine, 28(1):1-13. 
Table 1: Triage Category and Time in ED

\begin{tabular}{|c|c|c|c|c|c|c|}
\hline \multirow[t]{2}{*}{ Time in ED } & \multicolumn{6}{|c|}{ Triage Category } \\
\hline & 1 & 2 & 3 & 4 & 5 & Total \\
\hline \multirow[t]{2}{*}{$0-2$ hours } & 9 & 125 & 213 & 28 & 1 & 376 \\
\hline & $2.6 \%$ & $6.4 \%$ & $7.3 \%$ & $18.2 \%$ & $25.0 \%$ & $7.0 \%$ \\
\hline \multirow[t]{2}{*}{ 2.1-4 hours } & 96 & 876 & 1361 & 60 & 3 & 2396 \\
\hline & $28.0 \%$ & $45.2 \%$ & $46.5 \%$ & $39.0 \%$ & $75.0 \%$ & $44.7 \%$ \\
\hline \multirow[t]{2}{*}{ 4.1-6 hours } & 111 & 438 & 660 & 31 & 0 & 1240 \\
\hline & $32.4 \%$ & $22.6 \%$ & $22.6 \%$ & $20.1 \%$ & $0.0 \%$ & $23.1 \%$ \\
\hline \multirow[t]{2}{*}{$6.1-8$ hours } & 52 & 233 & 335 & 17 & 0 & 637 \\
\hline & $15.2 \%$ & $12.0 \%$ & $11.5 \%$ & $11.0 \%$ & $0.0 \%$ & $11.9 \%$ \\
\hline \multirow[t]{2}{*}{$>8$ hours } & 75 & 268 & 355 & 18 & 0 & 716 \\
\hline & $21.9 \%$ & $13.8 \%$ & $12.1 \%$ & $11.7 \%$ & $0.0 \%$ & $13.3 \%$ \\
\hline \multirow[t]{3}{*}{ Total } & 343 & 1940 & 2924 & 154 & 4 & 5365 \\
\hline & $100.0 \%$ & $100.0 \%$ & $100.0 \%$ & $100.0 \%$ & $100.0 \%$ & $100.0 \%$ \\
\hline & & Value & df & $\mathrm{P}$-value & & \\
\hline \multicolumn{2}{|c|}{ Pearson Chi-Square } & $107.592^{\mathrm{a}}$ & 16 & 0.000 & & \\
\hline \multicolumn{2}{|c|}{ Likelihood Ratio } & 101.935 & 16 & 0.000 & & \\
\hline \multirow[t]{2}{*}{ Referred by } & \multicolumn{6}{|c|}{ Triage Category } \\
\hline & 1 & 2 & 3 & 4 & 5 & Total \\
\hline \multirow{3}{*}{$\begin{array}{l}\text { General } \\
\text { Practitioners }\end{array}$} & 3 & 106 & 247 & 20 & 0 & 376 \\
\hline & & & & & & \\
\hline & $0.9 \%$ & $5.5 \%$ & $8.4 \%$ & $13.0 \%$ & $0.0 \%$ & $7.0 \%$ \\
\hline \multirow[t]{2}{*}{ Nursing home } & 34 & 90 & 191 & 7 & 0 & 322 \\
\hline & $9.9 \%$ & $4.6 \%$ & $6.5 \%$ & $4.5 \%$ & $0.0 \%$ & $6.0 \%$ \\
\hline \multirow[t]{2}{*}{ Other hospital } & 13 & 67 & 133 & 4 & 0 & 217 \\
\hline & $3.8 \%$ & $3.5 \%$ & $4.5 \%$ & $2.6 \%$ & $0.0 \%$ & $4.0 \%$ \\
\hline \multirow{2}{*}{$\begin{array}{l}\text { Self/ family/ } \\
\text { friend }\end{array}$} & 292 & 1667 & 2342 & 123 & 4 & 4428 \\
\hline & $85.1 \%$ & $85.9 \%$ & $80.1 \%$ & $79.9 \%$ & $100 . \%$ & $82.5 \%$ \\
\hline \multirow[t]{3}{*}{ Others } & 1 & 10 & 11 & 0 & 0 & 22 \\
\hline & $0.3 \%$ & $0.5 \%$ & $0.4 \%$ & $0.0 \%$ & $0.0 \%$ & $0.4 \%$ \\
\hline & 343 & 1940 & 2924 & 154 & 4 & 5365 \\
\hline \multicolumn{7}{|l|}{ Total } \\
\hline & $100.0 \%$ & $100.0 \%$ & $100.0 \%$ & $100.0 \%$ & $100.0 \%$ & $100.0 \%$ \\
\hline & & Value & $\mathrm{df}$ & $\mathrm{P}$-value & & \\
\hline \multicolumn{2}{|c|}{ Pearson Chi-Square } & $69.958^{a}$ & 16 & 0.000 & & \\
\hline \multicolumn{2}{|c|}{ Likelihood Ratio } & 80.042 & 16 & 0.000 & & \\
\hline
\end{tabular}


Table 2: Triage Category and Hospital Length of Stay

\begin{tabular}{|c|c|c|c|c|c|c|}
\hline Triage categories & None & 1 day & 2-3 days & 4-5 days & $>5$ days & Total \\
\hline 1 & $32.9 \%$ & $12.2 \%$ & $17.8 \%$ & $12.8 \%$ & $24.2 \%$ & $100.0 \%$ \\
\hline 2 & $29.3 \%$ & $19.6 \%$ & $18.9 \%$ & $13.2 \%$ & $18.9 \%$ & $100.0 \%$ \\
\hline 3 & $34.2 \%$ & $22.0 \%$ & $17.2 \%$ & $8.8 \%$ & $17.8 \%$ & $100.0 \%$ \\
\hline 4 & $57.8 \%$ & $13.6 \%$ & $12.3 \%$ & $3.2 \%$ & $13.0 \%$ & $100.0 \%$ \\
\hline 5 & $100.0 \%$ & & & & & $100.0 \%$ \\
\hline Total & $33.1 \%$ & $20.3 \%$ & $17.7 \%$ & $10.5 \%$ & $18.5 \%$ & $100.0 \%$ \\
\hline
\end{tabular}


Table 3: Total cost (AUD) of Emergency Department presentations and ambulance services (all types) for COPD patients (2016-2018)

\begin{tabular}{|c|c|c|c|c|c|c|c|}
\hline Total & 5,253 & 100 & $4,179,549$ & Total & 4,246 & 80.8 & $2,792,385$ \\
\hline 1 ED pres. & 2,161 & 41.14 & $1,719,399$ & $\mathrm{DD}$ & 1,176 & 27.7 & $1,004,304$ \\
\hline >1 ED pres. & 3,092 & 58.56 & $2,460,149$ & WM & 1,143 & 26.9 & 649,224 \\
\hline$>1$ ED pres. $(D D)$ & 717 & 23.20 & 570,481 & GC & 1,927 & 45.4 & $1,138,857$ \\
\hline$>1$ ED pres. $(W M)$ & 866 & 28.00 & 689,032 & & & & \\
\hline$>1$ ED pres. $(G C)$ & 1,509 & 48.80 & $1,200,635$ & & & & \\
\hline
\end{tabular}

Note: Pres= Presentation. Average cost of each ED presentation = AUD 795.65. Average cost of ambulance per person: Darling Downs $(\mathrm{DD})=$ AUD 854.00; Gold Coast (GC)= AUD 568; and West Moreton (WM) = AUD 591 (FY 2016-17, Department of Health, 2018) 
Table 4: Total cost (AUD) of hospital stay for COPD patients admitted through ED

\begin{tabular}{|c|c|c|c|c|c|c|c|c|c|}
\hline \multirow[t]{2}{*}{ Hospital } & \multirow{2}{*}{$\begin{array}{c}\text { Number } \\
\text { of patients }\end{array}$} & \multicolumn{3}{|c|}{ Cost of hospital length of stay (AUD) } & \multicolumn{5}{|c|}{ Length of stay (\%) } \\
\hline & & $\underset{\$}{\text { Mean cost }}$ & $\begin{array}{c}5 \% \\
\text { trimmed } \\
\text { mean cost }\end{array}$ & Total & $\begin{array}{l}>0 \text { to } \\
<2 \\
\text { days }\end{array}$ & $\begin{array}{c}\geq 2 \text { to } \\
<4 \\
\text { days }\end{array}$ & $\begin{array}{l}\geq 4 \text { to } \\
<6 \\
\text { days }\end{array}$ & $\begin{array}{l}\geq 6 \\
\text { days }\end{array}$ & Total \\
\hline \multicolumn{10}{|c|}{ Time period 2016-2018 } \\
\hline Combined & 5,079 & 8,297 & 6,932 & $42,142,474$ & 34.7 & 27.9 & 16.4 & 20.9 & 100 \\
\hline Toowoomba & 1,006 & 11,059 & 9,171 & $11,125,616$ & 29.9 & 26.8 & 14.5 & 28.2 & 100 \\
\hline Ipswich & 1,715 & 7,637 & 6,345 & $13,098,440$ & 35.3 & 29.4 & 17.4 & 17.9 & 100 \\
\hline Gold Coast & 2,358 & 7,598 & 6,456 & $17,918,418$ & 36.3 & 27.3 & 16.4 & 20.0 & 100 \\
\hline \multicolumn{10}{|c|}{ Time period SEP-2017 to SEP-2018 } \\
\hline Combined & 2,440 & 8,122 & 6,812 & $19,818,119$ & 34.8 & 29.2 & 15.6 & 20.5 & 100 \\
\hline Toowoomba & 484 & 10,389 & 8,780 & $5,058,643$ & 31.6 & 29.3 & 12.6 & 26.4 & 100 \\
\hline Ipswich & 823 & 7,345 & 6,067 & $6,045,107$ & 36.8 & 29.2 & 17.5 & 16.5 & 100 \\
\hline Gold Coast & 1,133 & 7,691 & 6,577 & $8,714,378$ & 34.7 & 29.1 & 15.4 & 20.7 & 100 \\
\hline
\end{tabular}

Note: Average cost of staying at hospital per day = AUD 2066. Only considering patients who came to ED and stayed at the hospital. Zero or none hospital days were omitted. There were some NULL values (not considered). 
Table 5: Factors affecting hospital length of stay for COPD patients (Generalised linear model (bivariate regression with a maximum likelihood estimate)

\begin{tabular}{lcccccc}
\hline Variable & B & Std. Error & Lower & Upper & Sig. & Df \\
\hline Age at Triage & 0.036 & 0.0052 & 0.026 & 0.046 & 0.000 & 1 \\
Stayed at ED & 0.116 & 0.0212 & 0.074 & 0.157 & 0.000 & 1 \\
Insurance status [public] & & & & & & \\
Private & -0.010 & 0.1627 & -0.329 & 0.308 & 0.949 & 1 \\
Country of birth [Australia] & & & & & \\
All other countries & & & & & \\
Diagnosis Code [Others] & 0.229 & 0.1329 & -0.032 & 0.489 & 0.085 & 1 \\
J44.9 & & & & & \\
J44.1 & -1.047 & 0.1558 & -1.352 & -0.741 & 0.000 & 1 \\
J44.0 & -0.218 & 0.1716 & -0.555 & 0.118 & 0.204 & 1 \\
Employment status [Employed] & 0.712 & 0.1443 & 0.429 & 0.994 & 0.000 & 1 \\
Pensioners & & & & & & \\
Others & 0.774 & 0.2563 & 0.271 & 1.276 & 0.003 & 1 \\
Triage category [Triage category 1] & 0.636 & 0.2182 & 0.209 & 1.064 & 0.004 & 1 \\
[Triage Category=5] & & & & & & \\
[Triage Category=4] & -3.697 & 2.0396 & -7.695 & 0.301 & 0.070 & 1 \\
[Triage Category=3] & -2.515 & 0.4049 & -3.309 & -1.722 & 0.000 & 1 \\
[Triage Category=2] & -1.386 & 0.2344 & -1.845 & -0.927 & 0.000 & 1 \\
\hline Nit Dep & -0.784 & 0.2401 & -1.255 & -0.314 & 0.001 & 1 \\
\hline
\end{tabular}

Note: Dependent variable is length of stay 